\title{
Energy Efficient K-Means Clustering Technique for Underwater Wireless Sensor Network
}

\author{
Sunpreet Kaur \\ Mtech Research Scholar \\ Dept of Computer Science and \\ Engineering, SGGSWU, \\ Fatehgarh Sahib, PUNJAB, INDIA
}

\author{
Vinay Bhardwaj \\ Assistant Professor, \\ Dept of Computer Science \\ and Engineering, SGGSWU, \\ Fatehgarh Sahib, PUNJAB, INDIA
}

\begin{abstract}
The communication range of underwater wireless sensor networks (UWSN) is limited by the underwater environment. Acoustic networks with huge number of sensors may have long communication range with appropriate protocols in literature. On the other hand, especially, the networks including small number of nodes have communication problems for long ranges. In energy constrained 3D underwater system environment it is essential to discover approaches to enhance the lifetime of the sensor nodes. Underwater sensors cannot utilize sunlight-based vitality to recharge the batteries. To challenge this problem, Multihop communication in underwater acoustic networks is a promising solution. In this study, a novel approach, Multihop Energy Efficient K-Means Clustering algorithm (MH-EKMC) is introduced and developed. The goal of this paper is to produce simulation results that would show the exhibitions of the proposed protocol for a given metric such as Network lifetime, No of dead nodes per round and total energy consumption. From the results, proposed protocol shows better performance for an energy-constrained network.
\end{abstract}

\section{Keywords}

Underwater Sensor Networks; K-Means Clustering; Energy Efficiency; Network Lifetime; Acoustic Communication

\section{INTRODUCTION}

In recent years, underwater wireless sensor network has emerged as a powerful strategy with a specific goal to find and exploit the harsh environment [6]. As electromagnetic waves do not spread well in ocean water, acoustic channel gives the most obvious medium to empower underwater communication. Underwater communication channel has described by three main considerations: - attenuation that relies on upon the signal frequency, multipath proliferation and low speed of sound $(1500 \mathrm{~m} / \mathrm{s})$.the transmission speed and transmission control heavily depends on the distance [5]. To increase lifetime, energy must be spared in each equipment and programming solution. In the harsh underwater environment, however, an underwater sensor node is powerless against disappointments or physical harms, which may affect ordinary system operation. Radio waves, electromagnetic waves and optical signals can be scattered many ways because of transmitted for long separations in underwater. These arrangements can be taken care of by numerous procedures.

example: - acoustic communication used to transmit information in a productive way. Multihop systems are required for underwater sensor networks since sensors devour high power and substitution of batteries [8].

\subsection{Types of sensor networks}

A wireless sensor network for the most part has next to zero infrastructures. It comprises of number of sensor nodes working collectively to monitor a zone to acquire information about the environment. Fig 1 shows the overview of wireless sensor network and application of underwater and terrestrial networks. Wireless sensor network is divided into terrestrial and underwater wireless sensor network. Localization, deployment, security and calibration are the main issues in terrestrial sensor networks. Underwater wireless sensor network has also some issues like more power consumption, path loss, bandwidth and localization. In terrestrial sensor network, radio waves are used to communicate from one sensor node to another. Radio waves are not suitable for long communication, therefore these are not suitable for underwater. For long communication acoustic waves are used. Wireless sensor networks can be classified based on the environment of applications [10].

\subsubsection{Terrestrial wireless sensor network}

Terrestrial wireless sensor network comprises of hundred of sensor nodes conveyed in either adhoc or preplanned way in an objective area. In terrestrial wireless sensor network, it is very easy to recharge and change the batteries because of solar energy. In terrestrial wireless sensor networks radio waves expends less energy than acoustic waves, which has utilized for underwater wireless sensor network.

\subsubsection{Underwater wireless sensor network:}

Underwater acoustic sensor network includes variable number of sensors and vehicle that conveys to perform community oriented observing errands over a given volume of mater. To transmit data from one sensor node to another node, acoustic signals are used in underwater wireless sensor network. In underwater wireless sensor network, energy consumption is more than the terrestrial wireless sensor network. 


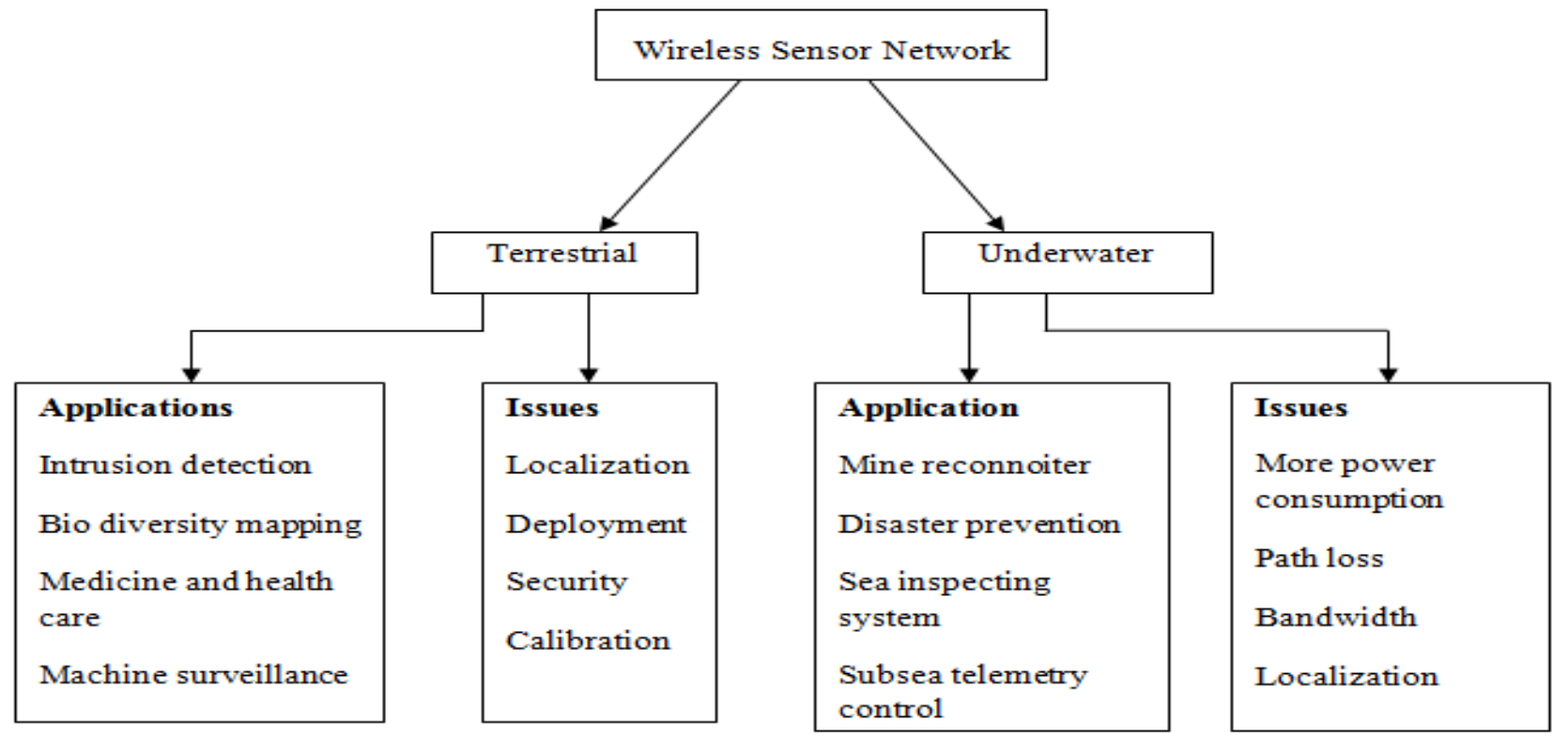

Figure 1: Overview of wireless sensor network

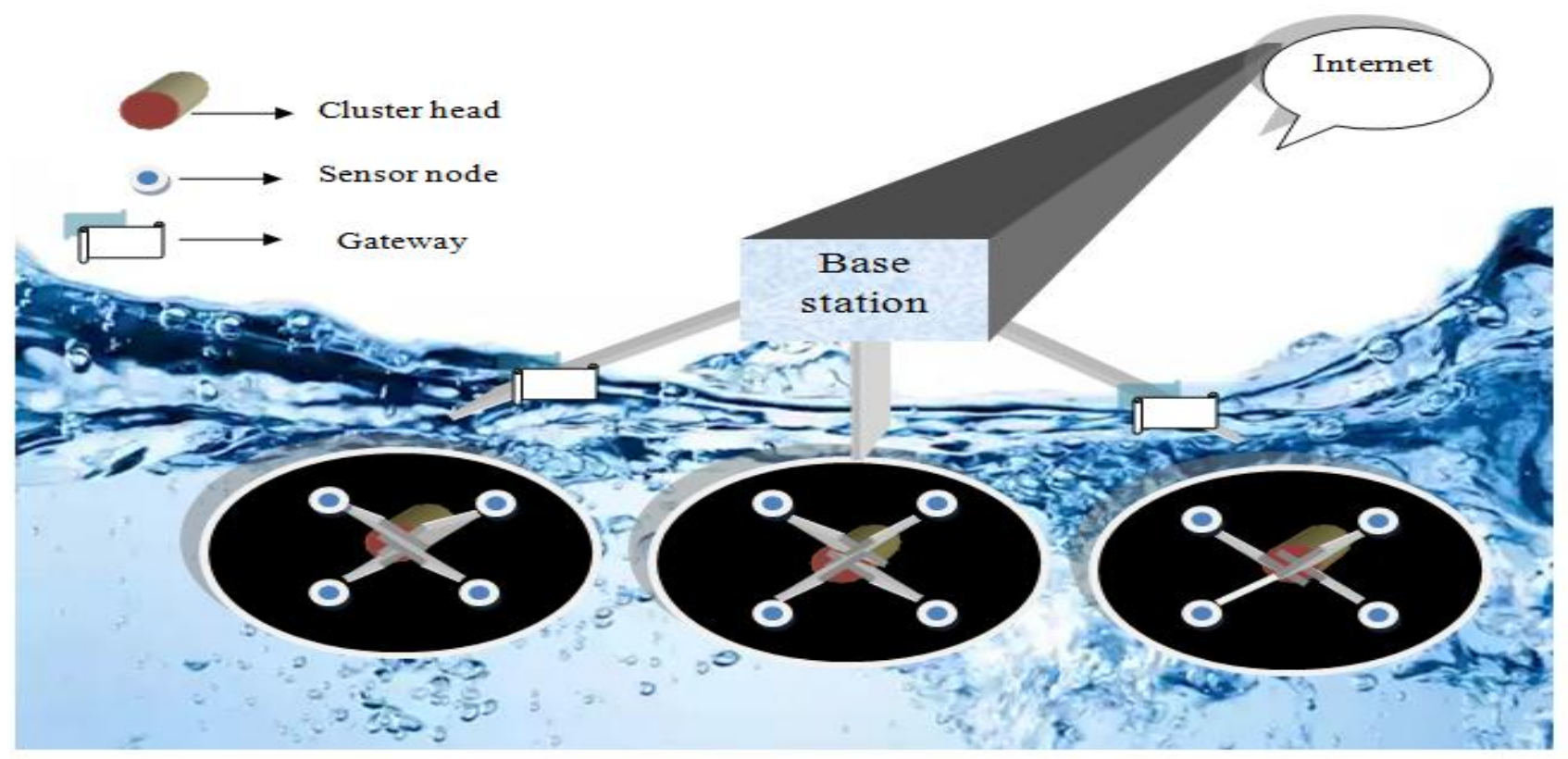

Figure 2: Proposed underwater network topology

\subsection{Architecture of Underwater sensor network}

Fig 2 describes the architecture of simple underwater wireless sensor network. This network contains four kinds of nodes, cluster head, base station, gateway nodes and sensor nodes. In this architecture, the entire system divides in to a few clusters. The ordinary sensors nodes used for the detecting task. The ordinary nodes cannot directly communication with the base station. Cluster heads are capable nodes that can assemble information from ordinary sensor. Cluster heads calculates distance from base station and gateway nodes. If distance from cluster head to base station is shorter, than cluster heads forward its data to the base station, otherwise data is forward to gateway nodes and these nodes forward data to base station [9].

\section{RELATED WORK}

LEACH [4] the first bunching steering protocol proposed for WSNs incorporates Low Energy Adaptive Clustering Hierarchy $(\mathrm{LEACH})$. In leach protocol set of sensor nodes are selected randomly as cluster head. Cluster setup and steady are two phases of LEACH protocol. In cluster setup phase cluster head is selected and then the cluster heads compress data that are gathered from other nodes. VBF (Vector-Based Forwarding Routing Protocol), Dina M. Ibrahim et al. [3] proposed Clustering Vector-Based Forwarding algorithm CVBF that divides the network into several cluster. Each cluster transmits the message following the mechanism of VBF. In this protocol, the boundary nodes cost too much energy, and it still has the problem of "Hotspots". DUCS protocol [7] is an improvement to this approach. It is used in non-time-critical aquatic monitoring applications in underwater environments. The basic 
properties of it are: simple, energy aware and GPS-free; it also minimizes the proactive routing exchange, uses data aggregation techniques and does not use flooding. Assuming random node mobility DUCS compensates the high propagation delays of the underwater medium using a continually adjusted timing advance combined with guard time values to minimize data loss. The combination of DUCS with TDMA/CDMA reduces interference and improves communication quality. MCCP [9] (Minimum Cost Clustering Protocol), the protocol depends upon expense metric. The cost metric is figured on the premise of three imperative parameters: (1) the all out energy utilization of the cluster individuals for sending information to the cluster head. (2) The lingering energy of the cluster head and its cluster members. (3) The relative area between the cluster head and the uw-sink. Depth Based Routing Protocol (DBR) [2] DBR does not require full dimensional area data of sensor nodes. It utilizes an avaricious way to deal with deliver packets to the sink at the water surface. It requires just nearby depth information, which can be effectively gotten with an inexpensive depth sensor that can be prepared in each underwater sensor node. REBAR [12] increases the reliability and effectiveness of data routing in the network. The source list figures a directional vector $\mathrm{v}$ from itself to sink, which is basically the steering course data. The Euclidean distance from source to sink $\mathrm{d}$ and the vector $\mathrm{v}$ are put away in the packet. The packet is relegated with a one of a unique ID, which is made out of the source ID and a sequence number. Every recipient keeps up a buffer to record the id of recently received packets. Duplicates can be inspected by the history and will be disposed of. Vector-Based Void Avoidance (VBVA) [13] extends the VBF to handle this problem. Initially, a routing vector is a path that sends data from source to target node. If void does not exist, VBVA acts the same as VBF. When there is a void, VBVA have tow mechanism, which are vector-shift mechanism or backpressure mechanism, to be utilized. KMeans algorithm is the most classic and widely used clustering algorithm based on partition, which belongs to the distancebased clustering methods [1]. It was proposed by J. B. Macqueen, and it is a very influential clustering technique in the industrial and scientific applications by far [11].

\section{ENERGY CONSUMPTION MODEL}

The energy model is used to calculate energy [14], which had proposed for underwater acoustic networks. According to this model, Tp denotes the transmitting time, A(d) denotes energy attenuation and denotes the distance.

$$
\begin{aligned}
& \mathrm{E}_{\mathrm{tx}}=\mathrm{P}_{\mathrm{r}} \times \mathrm{T}_{\mathrm{p}} \times \mathrm{A}(\mathrm{d}) \\
& \mathrm{T}_{\mathrm{p}=} \mathrm{M}_{\mathrm{b}} / \mathrm{S}_{\mathrm{v}}
\end{aligned}
$$

$\mathrm{Mb}$ denotes the size of the information packet

Sv is the transmission speed

$$
A(d)=d^{\lambda} \times \beta^{d}
$$

Where $\lambda$ is the spreading factor (1.5 for practical). The parameter $\beta=10^{\alpha(\mathrm{f}) / 10}$ is determined by the absorption coefficient $\alpha(\mathrm{f})$, which can be calculated by using following formula:

$$
\begin{gathered}
\alpha(\mathrm{f})=0.11 \times\left(10^{-3} \mathrm{f}^{2} / 1+\mathrm{f}^{2}\right)+44\left(10^{-3} \mathrm{f}^{2} / 4100+\mathrm{f}^{2}\right)+2.75 \times 10^{-7} \\
\mathrm{f}^{2}+3 \times 10^{-6}
\end{gathered}
$$

In this simulation, some assumptions are:

- Base station is remaining stationary all the time.

- All the sensor nodes spread over a three dimensional space.
- Both gateway nodes know the area of the base station.

- Each sensor node has same initial energy.

- All nodes measure the natural parameters and send it to the receiver node.

- Once every one of the nodes finish the data transmission to the base station, it is called as one round.

\section{THE PROPOSED ALGORITHM 4.1 Mutihop Energy Efficient K-Means Clustering Algorithm (MH-EKMC)}

In this algorithm, k-means clustering approach is used to form clusters. This algorithm has three phases: (1) cluster formation phase (2) Cluster head selection (3) Data transmission phase. In cluster formation phase, $\mathrm{k}$-means clustering method is used to form the clusters. In the cluster head phase clusters area selected randomly according to distance. In data transmission phase, data is transmitting to base station. In this phase the concept of two gateway nodes is added to collect, receive and transmission of data. In this phase, firstly, calculate the distance from all cluster heads to base station, gateway node 1 and gateway node 2 . Then the cluster heads checks the minimum distance. If the distance from cluster head to base station is minimum, $\mathrm{CHs}$ directly sends its data to base station. If the distance from cluster head to gateway node one and gateway node two is minimum than the cluster heads sends its data to gateway these gateway nodes and these gateway nodes are directly connected to base station to forward the data. Now evaluate performance on total energy consumption, first node dead, number of dead nodes and number of alive nodes. This algorithm shows better results than direct transmission.

\section{Algorithm:}

Step 1: Cluster formation

Apply k-means algorithm to form clusters

- Each cluster $\mathrm{k}(\mathrm{i})$ contains a number of nodes $\mathrm{i}=1$ to $\mathrm{n}$

- Initially all nodes have the same energy.

Step 2: Cluster head selection

- $\quad$ Select $\mathrm{k}$ initial cluster heads

- Nodes join in the nearest cluster

Step 3: Data transmission

- Calculate distance from cluster heads to BS, GT1 (Gateway 1), and GT2 (Gateway 2).

- If distance from CHS to BS is minimum than send data to BS, otherwise send it to GT1, GT2.

\section{SIMULATION AND DISCUSSION}

In this section, the execution of proposed algorithms through broad simulations under MATLAB is evaluated. An extensive set of simulation has carried out with fixed frequency $3.4 \mathrm{kHz}$ for 100 no of nodes. A set of simulation has performed for the selection of the parameters. Receiving power to decode the packet is fixed in this simulation. The simulation parameters are listed below in the table: 
Table 1: Simulation parameters

\begin{tabular}{|c|c|}
\hline Parameters & Values \\
\hline Initial Energy & $10 \mathrm{~J}$ \\
\hline Network Size & $100 \times 100 \times 100 \mathrm{~m}^{3}$ \\
\hline Number of Nodes & 100 \\
\hline $\begin{array}{c}\text { Number of Gateway } \\
\text { Nodes }\end{array}$ & 2 \\
\hline Etx & $0.0271 \mathrm{~J}$ \\
\hline Erx & $0.0090 \mathrm{~J}$ \\
\hline Communication Model & Acoustic \\
\hline
\end{tabular}

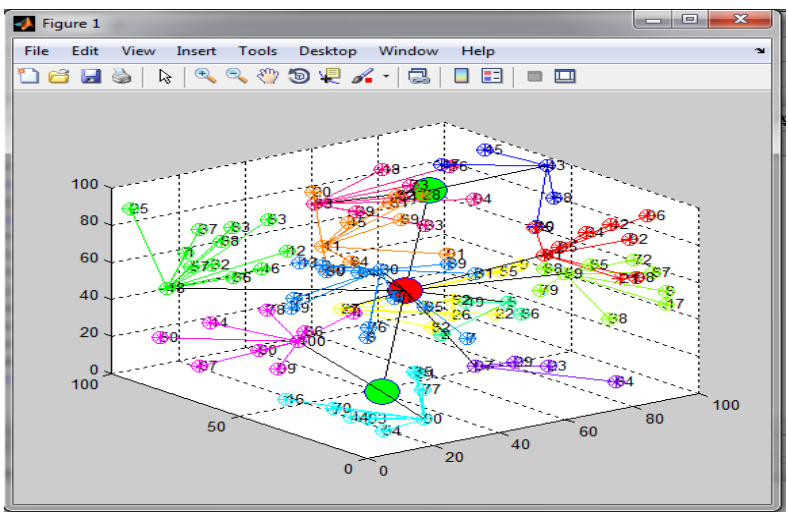

Figure 3: Deployment of nodes in 3-D Space

Figure 3 shows the deployment of nodes in three dimensional space. In this figure 100 nodes has deployed with area $(100 \times 100 \times 100)$. Base stattion is placed with cordinates $(50 \times 50 \times 150)$.

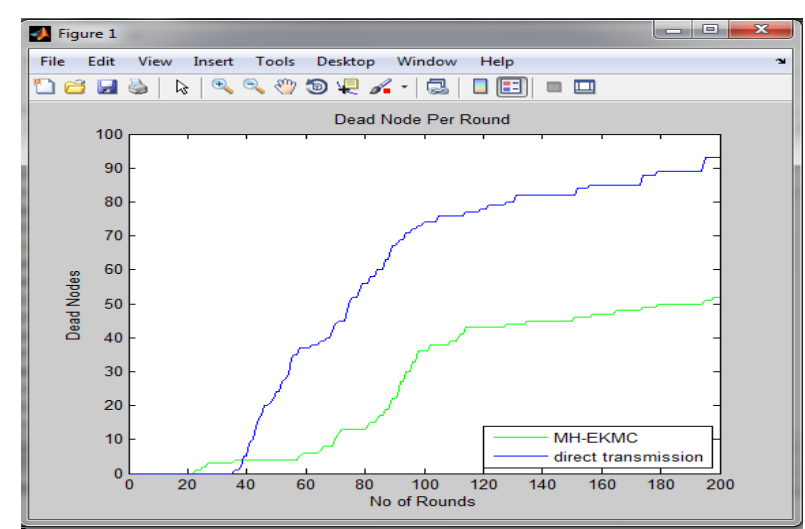

Figure 4: Dead nodes vs. No of rounds

In Figure 4, In MH-EKMC algorithm 52 nodes has dead in 200 rounds and in direct transmission 95 nodes has dead in 200 rounds. Therefore, in this set of simulations, the MH-EKMC algorithm is $40 \%$ more efficient as compared to the direct transmission

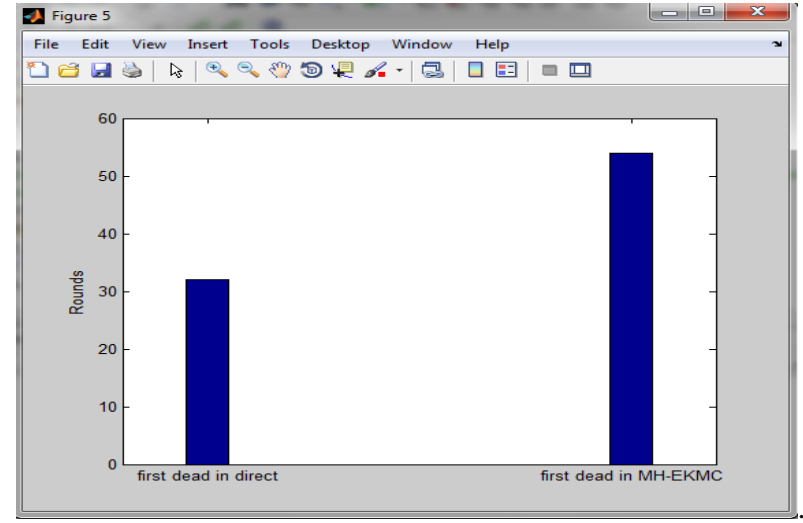

Figure 5: First node dead vs. No of rounds

In Figure 5, the first node dies in direct transmission algorithm after 33 rounds. In MH-EKMC, first node dies after 53 rounds. In this simulation, the MH-EKMC is more efficient in terms of network lifetime as compared to direct transmission.

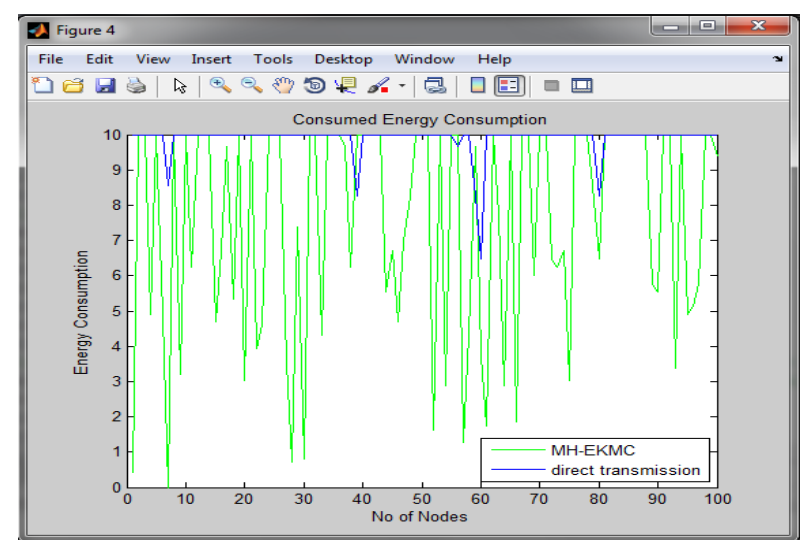

Figure 6: Comparison of total energy consumption

In Figure 6, the total energy consumption of proposed algorithm and a comparison with direct transmission has been performed. As shown in this figure, the MH-EKMC algorithm consumes less energy as compare to direct transmission.

\section{CONCLUSION}

A large portion of the proposed algorithms were just connected to two-dimensional systems where all sensor nodes are dispersed in a two dimensional plane (2D). In this research, a few two-dimensional (2D) geometric topologies are extended to three-dimensional plane (3D).The main objective of this paper is to design routing protocol for underwater sensor network that will increase the energy efficiency of overall network. In this work, multihop technique in MH-EKMC algorithm is applied for underwater wireless sensor network by considering some parameters like first node dead, last node dead, dead nodes per round and energy consumption per round by which the lifetime of the overall network is improved.

\section{REFERENCES}

[1] Elhamifar E, Vidal R. Sparse subspace clustering: Algorithm, theory, and applications. Pattern Analysis and Machine Intelligence, IEEE Transactions on, vol. 35, no.11, pp. 2765-2781, 2013

[2] Hai Yan, Zhijie Jerry Shi, and Jun-Hong Cui "DepthBased Routing for Underwater Sensor Networks" Department of Computer Science and Engineering University of Connecticut, Storrs, CT 06269-2155. 
[3] Ibrahim D M, Eltobely T E, Fahmy M M, et al. Enhancing the Vector-Based Forwarding Routing Protocol for Underwater Wireless Sensor Networks: A Clustering Approach. ICWMC 2014, The Tenth International Conference on Wireless and Mobile Communications. pp. 98-104, 2014.

[4] Lalita Yadav et al, "Low Energy Adaptive Clustering Hierarchy in Wireless Sensor Network (LEACH)" (IJCSIT) International Journal of Computer Science and Information Technologies, Vol. 5 , 4661-4664, 2014.

[5] Mander Chitre, Shiraz Shahabudeen, "Recent advances in underwater acoustic communication \& networking", International journal of soft computing , 2000.

[6] Manijeh Keshtgary, Reza Mohammadi "Energy Consumption Estimation in Cluster based Underwater Wireless Sensor Networks Using M/M/1 Queuing Model "International Journal of Computer Applications (0975 8887) Vol-43, No.24, April 2012.

[7] Mari Carmen Domingo,1 Rui Prior. "A Distributed clustering scheme for underwater wireless sensor networks", The 18th Annual IEEE International Symposium on Personal, Indoor and Mobile Radio Communications (PIMRC'07) .

[8] Ms. Kalpana .S, Mr. Arutchelvan G "An Accoustic Sensor for Transmission in Underwater Environment International Research Journal of Engineering and Technology (IRJET), Vol-2,Aug-2015
[9] Sihem Souiki1, Mourad Hadjila1 and Mohammed Feham1"Fuzzzy Based Clustering And Energy Efficient Routing For Underwater Wireless Sensor Networks" International Journal of Computer Networks \& Communications (IJCNC) Vol.7, No.2, March 2015.

[10] Syed Abdul Basit , Manoj Kumar "A Review of Routing Protocols for Underwater Wireless Sensor Networks" IJARCCE, Vol. 4, Issue 12, December 2015.

[11] Wang W, Yang J, Muntz R. STING: A statistical information grid approach to spatial data mining. VLDB. vol. 97, pp. 186-195, 1997.

[12] Xiaobing Wu , Guihai Chen “ A reliable and energy balanced routing algorithm for UWSNs" State Key Laboratory for Novel Software Technology Nanjing University ,Nov 2008.

[13] Xie P., Zhou Z., Peng Z., Cui J.-H., and Shi Z , "Void Avoidance in Three-dimensional Mobile Underwater Sensor Networks", Proc. of the 4th international conference of wireless algorithms, system, and applications (WASA ), USA, 2009.

[14] Peng Jiang, Jun Liu, Feng Wu , Jianzhong Wang and Anke Xue "Node Deployment Algorithm for Underwater Sensor Networks Based on Connected Dominating Set", Vol- 16, 2010. 